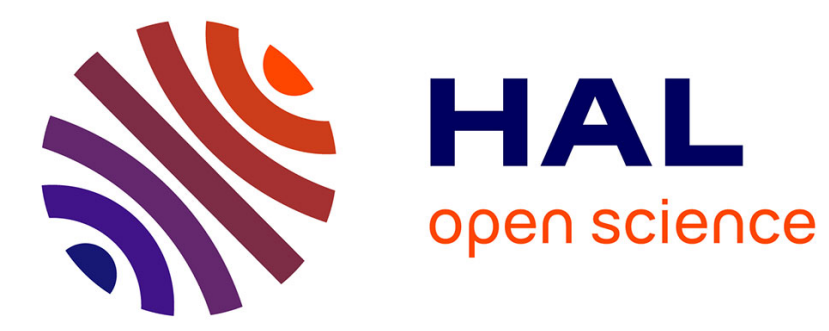

\title{
The burden of chronic kidney disease in systemic lupus erythematosus: A nationwide epidemiologic study
}

Arthur Mageau, Jean-François Timsit, Anne Perrozziello, Stéphane Ruckly, Claire Dupuis, Lila Bouadma, Thomas Papo, Karim Sacre

\section{To cite this version:}

Arthur Mageau, Jean-François Timsit, Anne Perrozziello, Stéphane Ruckly, Claire Dupuis, et al.. The burden of chronic kidney disease in systemic lupus erythematosus: A nationwide epidemiologic study. Autoimmunity Reviews, 2019, 18, pp.733 - 737. 10.1016/j.autrev.2019.05.011 . hal-03484458

\author{
HAL Id: hal-03484458 \\ https://hal.science/hal-03484458
}

Submitted on 21 Dec 2021

HAL is a multi-disciplinary open access archive for the deposit and dissemination of scientific research documents, whether they are published or not. The documents may come from teaching and research institutions in France or abroad, or from public or private research centers.
L'archive ouverte pluridisciplinaire HAL, est destinée au dépôt et à la diffusion de documents scientifiques de niveau recherche, publiés ou non, émanant des établissements d'enseignement et de recherche français ou étrangers, des laboratoires publics ou privés.

\section{(ㅇ)(1) $\$$}

Distributed under a Creative Commons Attribution - NonCommerciall 4.0 International 


\title{
Aberrant expression of alternative splicing variants in multiple sclerosis - a systematic review
}

Michael Hecker ${ }^{\mathrm{a},{ }^{\star}}$, Annelen Rüge ${ }^{\mathrm{a}, \S}$, Elena Putscher ${ }^{\mathrm{a}, \S}$, Nina Boxberger ${ }^{\mathrm{a}}$, Paulus Stefan Rommer ${ }^{\mathrm{a}, \mathrm{b}}$, Brit Fitzner ${ }^{a}$, Uwe Klaus Zettl ${ }^{a}$

\author{
a University of Rostock, Department of Neurology, Division of Neuroimmunology, \\ Gehlsheimer Str. 20, 18147 Rostock, Germany \\ ${ }^{\mathrm{b}}$ Medical University of Vienna, Department of Neurology, \\ Währinger Gürtel 18-20, 1090 Vienna, Austria
}

$\S$ these authors contributed equally to this work

*corresponding author: Dr. Michael Hecker, e-mail: michael.hecker@rocketmail.com, phone: +49 381 494-5890, fax: +49 $381494-5882$

Running title: Alternative pre-mRNA splicing in MS 


\section{Highlights}

- We identified 36 studies that investigated alternatively spliced gene isoforms in MS

- Splice variants were examined for 27 genes, e.g. immune-related and myelin genes

- An MS-associated skipping of $I L 7 R$ exon 6 has been validated at mRNA and protein level

- Transcriptome data are increasingly utilized for the analysis of RNA splicing

- The individual clinical implications of aberrant splicing events remain to be explored 


\section{Abstract}

OBJECTIVE: Alternative splicing is an important form of RNA processing that affects nearly all human genes. The differential expression of specific transcript and protein isoforms holds the potential of novel biomarkers for complex diseases. In this systematic review, we compiled the existing literature on aberrant alternative splicing events in multiple sclerosis (MS).

METHODS: A systematic literature search in the PubMed database was carried out and supplemented by screening the reference lists of the identified articles. We selected only MSrelated original research studies which compared the levels of different isoforms of human protein-coding genes. A narrative synthesis of the research findings was conducted. Additionally, we performed a case-control analysis using high-density transcriptome microarray data to reevaluate the genes that were examined in the reviewed studies.

RESULTS: A total of 160 records were screened. Of those, 36 studies from the last two decades were included. Most commonly, peripheral blood samples were analyzed (32 studies), and PCR-based techniques were usually employed (27 studies) for measuring the expression of selected genes. Two studies used an exploratory genome-wide approach. Overall, 27 alternatively spliced genes were investigated. Nine of these genes appeared in at least two studies (CD40, CFLAR, FOXP3, IFNAR2, IL7R, MOG, PTPRC, SP140 and TNFRSF1A). The microarray data analysis confirmed differential alternative pre-mRNA splicing for 19 genes.

CONCLUSIONS: An altered RNA processing of genes mediating immune signaling pathways has been repeatedly implicated in MS. The analysis of individual exon-level expression patterns is stimulated by the advancement of transcriptome profiling technologies. In particular, the examination of genes encoded in MS-associated genetic regions may provide important insights into the pathogenesis of the disease and help to identify new biomarkers.

Keywords: multiple sclerosis, alternative splicing, systematic review, RNA processing, gene isoforms, blood biomarkers, autoimmunity, transcriptomics, genetics, IL7R 


\section{Introduction}

Multiple sclerosis (MS) is a chronic inflammatory disease of the central nervous system (CNS) that leads to demyelination, neuroaxonal degeneration, synaptic loss and gliosis [1]. Typical symptoms include vision problems, limb weakness, sensory loss, cognitive impairment, fatigue, pain and muscle spasms [1]. More than 2.3 million people worldwide suffer from MS [2]. The average age at diagnosis is between 20 and 40 years [3]. Different courses of MS are distinguished: Approximately $85 \%$ of the patients are diagnosed with relapsing-remitting MS (RRMS), and $15 \%$ have a primary progressive course of MS (PPMS) from onset $[4,5]$. In about $60 \%$ of the RRMS patients, the disease turns after approximately 20 years into a secondary progressive MS (SPMS) [6].

The management of MS includes the treatment of acute relapses with glucocorticosteroids [7, 8] as well as symptomatic [9] and preventive therapies [10]. Within the past 20 years, more than 10 disease-modifying drugs (DMD) were approved for MS. The major mechanisms of action of DMD include immunomodulation (e.g. interferon- $\beta$ and glatiramer acetate), alteration of cell trafficking (e.g. natalizumab and fingolimod) and immune cell depletion (e.g. alemtuzumab and ocrelizumab) [10]. A first-line therapy for RRMS is subcutaneously or intramuscularly administered interferon- $\beta$ (IFN- $\beta$ ) [11]. A DMD for more active RRMS is fingolimod, an orally administered sphingosine-1-phosphate receptor modulator that prevents the migration of immune cells from lymph nodes into the peripheral blood, thereby reducing the annual relapse rate up to $69 \%$ [12]. More recently, intravenous infusions of ocrelizumab, a monoclonal antibody depleting CD20-expressing B cells, have been approved for the treatment of PPMS, given a $25 \%$ relative risk reduction in 24-week confirmed disability progression [13].

The etiology of MS is still not clear. Environmental factors like smoking, vitamin D deficiency and viral infections, for example with Epstein-Barr virus, have been associated with an increased susceptibility to MS [14-16]. In twin studies, a concordance rate for monozygotic twins of approximately $20 \%$ could be observed, suggesting a genetic component of MS [17, 18]. The largest genome-wide association study (GWAS) identified 233 genetic loci that are linked to MS risk [19]. More than $90 \%$ of the respective lead single-nucleotide polymorphisms 
(SNP) are located in non-coding regions, with the underlying mechanisms being largely unknown. However, it is likely that intergenic and intronic MS-associated SNPs may have an effect on transcription and RNA processing, e.g. alternative splicing.

Splicing is a physiological process in the cell nucleus that coordinates the excision of noncoding regions (introns) and the assembly of expressed regions (exons) of RNA molecules by a large ribonucleoprotein complex, the spliceosome [20] (Figure 1A). Cis-regulatory elements like the 5' and 3' splice sites (donor and acceptor), the branchpoint sequence (BPS) and the polypyrimidine tract (PPT) represent important recognition sequences for the processing of RNA [21-23]. Furthermore, trans-acting splice factors such as SR proteins may have either enhancer or silencer functions [24]. The differential use of multiple splice sites offers the possibility to create different mRNAs from one pre-mRNA. Therefore, alternative splicing events (ASE), together with alternative transcription start and termination sites, lead to a great transcriptome diversity. The basic types of ASE are exon skipping, intron retention, alternative splice site selection and utilization of mutually exclusive exons (Figure 1B). Some genetic variants cause an alteration of splicing regulation and thereby lead to different diseases [25]. Splice-switching antisense oligonucleotides that are used in the treatment of monogenic neuromuscular disorders, e.g. spinal muscular atrophy and Duchenne muscular dystrophy, demonstrate that an effective intervention in alternative RNA splicing is possible [26].

Improved methods foster the elucidation of disease-specific ASE. It is now possible to profile the spliceo-transcriptome via microarrays and high-throughput sequencing as two important approaches for genome-wide analyses [27, 28]. While microarrays are based on short oligonucleotides derived for annotated transcripts, high-throughput sequencing produces short reads without prior knowledge on the sequence. Driven by advancements in technology and bioinformatics, the investigation of alternative splicing gained more and more attention in recent years $[29,30]$. The characterization of ASE could support the search for biomarkers for the diagnosis, prognosis and monitoring of MS. The vast majority of research so far focused on the analysis of blood and cerebrospinal fluid (CSF) samples of MS patients with the aim to detect molecular biomarkers with high specificity and sensitivity [31, 32]. However, continued validation and testing is required before such biomarkers can be applied in clinical practice. 
Here, we reviewed the literature for studies that investigated alternative splicing in the context of MS, and we show how transcriptome microarray data can provide insights at the exon level of gene transcripts. We discuss that a better understanding of the regulation of gene isoforms is helpful in elucidating the pathomechanisms of MS and in identifying novel biomarkers. 


\section{Material and methods}

\subsection{Search strategy and study selection}

Studies for this systematic review were identified through the electronic database PubMed using the specific search terms "splicing" and "multiple sclerosis". The last update of the database search was performed on March 20, 2019. There was no restriction on the date of publication. Additionally, further articles, which were considered to be relevant from scanning the reference lists of the identified articles, were also included in the study selection process.

We considered the Preferred Reporting Items for Systematic Reviews and Meta-Analyses (PRISMA) that were developed by an international group of researchers [33]. Accordingly, we applied the four steps "identification“, "screening", "eligibility" and "inclusion". In the screening, only studies whose full text was published in English in a scientific journal were included. In the first part of the "eligibility" step, records were excluded if they presented no original research. Next, the articles were checked if at least two different splicing isoforms of a human proteincoding gene were measured experimentally. Finally, we screened the remaining articles for whether samples from MS patients were investigated. There was no restriction on the sample material, the examined cells or the used technologies. Moreover, no restrictions concerning disease duration, severity of disease, MS course or the treatment of patients were applied. Eligibility assessment was carried out by two reviewers (AR and EP) in accordance with the PRISMA workflow. Any discrepancies were resolved by discussion with a third reviewer $(\mathrm{MH})$.

\subsection{Data extraction}

To compare the included studies, a table providing detailed information for each study was created. For each study, we gathered the information which sample material was used (e.g. blood cells), which detection methods were used (e.g. polymerase chain reaction, PCR), whether genotypes were examined and whether a splicing reporter minigene assay was used [34]. Furthermore, we ascertained the study design (e.g. comparison of MS cases vs. controls or treated vs. untreated subjects) of the individual records and whether significant differences were reported between the groups. 
For the genes and ASE that were investigated in the included studies, a second table was created. As some of the examined genes with alternative splice variants were named by aliases in the studies, e.g. CD45 for PTPRC, the official gene symbols of the HUGO Gene Nomenclature Committee (HGNC) were used for standardization. The GeneCards database Suite 4.9 was then used to retrieve integrated gene-centric information from several external databases [35]. For each gene, the identification number for the UniProt database release 2019_02 as well as the respective count of annotated protein isoforms were determined [36]. Additionally, we extracted the numbers of transcript variants and the gene identifiers according to Ensembl database release 95 [37].

\subsection{Microarray datasets}

To reanalyze at exon level the genes for which MS-specific ASE have been described in the literature, we used a transcriptome dataset that was previously published by our research group (GEO database SuperSeries accession number GSE73174) [38-41]. For this dataset, 150 high-density GeneChip Human Transcriptome Arrays (HTA) 2.0 with six million 25mer oligonucleotide probes per array were used to study gene expression shifts in response to fingolimod therapy in patients with MS. Peripheral blood samples were taken to isolate 5 different cell populations (CD4+, CD8+, CD14+, CD19+ and CD56+ cells) for 10 RRMS patients at 3 different time points (before the start of therapy, one day after the first dose of fingolimod as well as 3 months after treatment initiation). Details on extraction and processing of RNA as well as on hybridization and scanning of the Affymetrix HTA 2.0 microarrays are described elsewhere [41]. Here, we used a subset of 100 arrays and discarded the 3-month time point for which we observed strong therapy-induced transcriptome changes [40].

To realize a case-control analysis, we then compared our dataset for MS patients to public HTA 2.0 microarray datasets for healthy subjects. Four suitable datasets were found in the GEO database: GSE63379 ( $n=32$ arrays for peripheral blood mononuclear cells, PBMC), GSE88887 [42] ( $n=60$ arrays for whole blood samples) and two subsets of GSE111555 ( $n=84$ arrays for whole blood samples and $n=32$ arrays for PBMC). In total, the 4 control datasets comprised 208 microarrays, yielding high-resolution transcriptome profiles of human blood cells. 


\subsection{Statistical evaluation}

The HTA 2.0 microarrays are designed with 10 probes per exon fragment (PSR) and 4 probes per exon-exon splice junction (JUC), facilitating a deep view into alternative splicing patterns. The own and external raw data (308 CEL files in total) were processed in one run with the Transcriptome Analysis Console (TAC) 4.0.1 software, which is freely available from Thermo Fisher Scientific. The default settings of the program were used (e.g. SST-RMA normalization). For the ASE analysis, we employed the Splicing Index and the EventPointer algorithm [43] as implemented in the TAC software. By this means, the exon-level expression data of our MS patients (split into 5 immune cell populations) were compared against those of the 4 control groups. A gene was considered as differentially alternatively spliced if the previously reported ASE was consistently found with EventPointer $p$-value $<0.0001$ in all 4 comparisons. 


\section{Results}

\subsection{Study selection}

Through PubMed database searching and reference list scanning, the literature search resulted in a sum of 160 articles. The flow diagram of the study selection process according to the PRISMA statement [33] is presented in Figure 2. Three of the 160 articles were excluded because the full texts were not published in the English language. A total of 121 articles were ineligible for the following reasons: no original research (e.g. reviews) ( $n=32)$, no investigation of at least two different splicing isoforms of human protein-coding genes $(n=47)$ and no examination of sample material from MS patients $(n=42)$. In the end, 36 studies were included in this systematic review [44-79].

\subsection{Assessment of the included studies}

For the qualitative synthesis of the 36 selected articles, we extracted information on the investigated genes, the type of sample material, the laboratory methods and the study design (Table 1). Almost all studies $(n=34)$ evaluated only 1-3 different genes. The exceptions were the microarray investigation by Tian et al., in which 92 different alternatively spliced genes were identified [67], and the recent RNA sequencing analysis by Cardamone et al., in which 957 alternatively spliced genes (1114 ASE) were identified [78]. The first articles in this research field were published in the year $2000[50,53]$. While only 10 articles were published until 2007, the number of published articles increased by a factor of $>3$ within the last years. The studies explored disease mechanisms and the informative value of ASE with regard to the diagnosis and therapy of MS. The comparison of MS cases and controls was the most common study design ( $n=31$ ). Accordingly, the aberrant expression of specific mRNA splicing isoforms has been discussed as potential disease-specific biomarkers. Several studies $(n=20)$ investigated genotype-dependent ASE, and 7 studies examined the effect of IFN- $\beta$ treatment on gene isoform levels in blood cells. Two of these studies additionally included blood samples from patients treated with immunosuppressive agents and glatiramer acetate, respectively [59, 68]. The research groups by Gilli et al. and López-Gómez et al. analyzed the long-term effect of IFN- $\beta$ injections by comparing RNA isoform levels in blood cells from MS patients before and 
up to 2 years after the start of IFN- $\beta$ treatment $[69,74]$. The size of the analyzed MS cohorts varied from 5 patients to more than 1000 patients [44, 48].

Most commonly, PCR assays were utilized for the measurement of gene isoforms $(n=27$ studies). Cardamone et al. additionally performed RNA sequencing using an Illumina NextSeq 500 platform to determine ASE that are modulated by MALAT1, a non-coding RNA that is expressed at higher levels in MS patients and involved in the regulation of the pre-mRNA splicing machinery [78]. Tian et al. was the only research group that used Affymetrix GeneChip Exon 1.0 ST Arrays to identify significant differences in the alternative splicing of transcripts distinguishing untreated MS patients and healthy controls [67]. Of the 957 genes filtered by Cardamone et al. [78] and the 92 genes filtered by Tian et al. [67], only 3 genes (DDX39B, IFNAR2 and NFAT5) were examined in the other included studies. Six studies included experiments with a splicing reporter minigene spanning at least one exon and the flanking intronic sequences. The study by Gregory et al., for instance, was the first demonstrating via transfections of minigene constructs that the MS-associated SNP rs6897932 affects the inclusion of exon 6 of $I L 7 R[44]$.

Blood cells, e.g. whole blood samples or PBMC, have been examined in 32 studies and, therefore, they were the most widely used sample material. In 24 of these studies, transcript isoform expression was examined with PCR-based methods or microarrays. Protein isoform levels, on the other hand, were determined in 19 of these studies, e.g. with flow cytometry, enzyme-linked immunosorbent assays (ELISA) or immunoblots. Specific immune cell types, e.g. T cells, B cells or dendritic cells, were analyzed in a total of 16 studies. Taking into account that MS is an inflammatory disease of the CNS, we found that a total of 5 studies used postmortem brain tissues or CSF samples for the analysis. Ten studies compared alternative splicing expression patterns among different cells and tissues. For instance, Boyle et al. explored the subcellular localization of MOG protein isoforms using confocal microscopy and the expression of the respective RNA variants in brain and other tissues using PCR followed by gel electrophoresis [55]. All studies that investigated ASE in cell culture conditions $(n=15)$ employed human cell lines, e.g. HEK 293, HeLa or Jurkat. 
A subset of 3 studies compared gene isoform levels in sample material from patients with different MS courses $[45,50,52]$. McKay et al. determined the ratio of soluble to full-length IL7R mRNA in the blood of patients with PPMS and RRMS [45]. In two studies by Sharief et al., the levels of the long and short protein isoforms of CFLAR were investigated in the blood of RRMS and SPMS patients [50,52]. In another study by this group, CFLAR isoform expression was compared between clinically active and clinically stable RRMS patients [51].

\subsection{Investigated alternative splicing events related to MS}

Altogether, 27 different genes have been explicitly studied in the 36 articles (Table 2). The most studied gene is the cytokine receptor ILTR that plays an important role in the differentiation and proliferation of human T cells. A soluble form of $I L 7 R$ results from an alternative splicing of exon 6. In 2007, Gregory et al. discovered the C allele of SNP rs6897932 in exon 6 as a significant risk factor for MS susceptibility in four independent family-based and case-control datasets [44]. They cloned the two allelic versions of this SNP in splicing reporter minigenes and observed a twofold increase of exon 6 skipping for the MS-associated C allele compared with the T allele. In a follow-up study from 2017, the same research group elucidated cis-acting elements and trans-acting factors controlling splicing of this exon [49]. They showed that the knockdown of the RNA helicase $D D X 39 B$ leads to a significantly higher level of exon 6 skipping and an increased secretion of soluble $I L 7 R$. The study by Hoe et al. is the only one that examined IL7R isoforms at the protein level in sera from MS patients and controls [47]. They confirmed that carriers of the T allele of SNP rs6897932 have significantly lower levels of soluble $I L 7 R$, and there was a non-significant trend toward higher levels in the MS patient group compared with healthy controls.

For the reason that MOG is a target in inflammatory demyelinating diseases [80], Jensen et al. used brain tissue specimens to determine relative levels of $M O G$ transcript variants for different haplotypes [56]. They observed a significant expression difference of transcripts resulting from two alternative splice acceptor sites of the last exon 10, which was related to the alleles of SNP rs2857766. While this study was focused on the influence of genetic variants on splicing, Boyle et al. evaluated 6 different $M O G$ isoforms with regard to their cellular localization by cloning 
them into mammalian expression vectors [55]. They showed that while the two full-length isoforms are expressed at the cell surface, three alternatively spliced isoforms characterized by exon 8 skipping have a more intracellular distribution. Another MOG isoform, an alternative last exon variant which lacks a transmembrane domain, is preferentially secreted. All six isoforms were detectable in normal appearing white matter from MS patients, but the MOG splicing pattern was not different compared to brain tissue from normal controls.

Most of the genes for which ASE have been investigated in the MS literature are involved in immune responses. Some of them encode cytokine receptors. Beyond IL7R, these are IFNAR2, CSF1R and members of the TNF receptor superfamily. The functional consequence of an MS-associated genetic variant in TNFRSF1A was investigated by two research groups $[59,60]$. They showed that the putative causal risk allele G of SNP rs 1800693 promotes exon 6 skipping and thus directs the expression of a soluble form that lacks the transmembrane and cytoplasmic domains and that can perturb TNF-a signaling. Splicing isoforms of the type I interferon receptor IFNAR2 modulate the responsiveness to both endogenous and recombinant IFN- $\beta$. Gilli et al. demonstrated that the transcript levels of the soluble form (exon 8 skipped) are significantly upregulated in PBMC of MS patients over the first year of IFN- $\beta$ therapy [69]. CSF1R is the receptor for CSF1, a cytokine which regulates monocyte/macrophage differentiation. In the study by Spurlock et al., a decreased transcript abundance of CSF1R exon 1-11 was observed in the blood of MS patients compared to healthy controls [65].

Several of the alternatively spliced genes control transcriptional programs. For instance, SP140 regulates the expression of immune-related genes that are linked to MS and other autoimmune diseases [81]. GWAS have identified the SP140 gene locus to be associated with the risk of developing MS [19], and Matesanz et al. could show that the T allele of SNP rs28445040 causes skipping of exon 7 in SP140 [64]. FOXP3 and NFAT5 also encode proteins that influence transcription. Matarese et al. found that regulatory $T$ cells (Treg) cells from patients with RRMS express lower amounts of the 44- and 47-kDa FOXP3 isoforms (resulting from exon 3 skipping/inclusion) as compared with Treg from controls, and they uncovered a link between glycolysis and FOXP3 pre-mRNA splicing via ENO1, a mechanism that may affect the induction and suppressive function of Treg $[57,58]$. NFAT5 regulates osmotic stress-related 
genes and is involved in high salt-induced Th17 cell development [82]. A splicing pattern analysis by Paraboschi et al. revealed that the levels of an exon 2-skipped NFAT5 isoform are significantly increased in MS cases vs. controls [66]. The inclusion of exon 2 depends on the genotype of SNP rs12599391, which is located within an intronic splicing enhancer (ISE). Interestingly, NFAT5 is activated by the Th17-specific protein kinase PRKCA [83], for which an MS-associated genetic polymorphism (rs35476409) was shown to affect exon 4 inclusion [63].

\subsection{Detection of alternative splicing events in transcriptome data}

The 36 included articles distinguished splicing variants of 27 different human protein-coding genes. The lack of validation studies prompted us to reevaluate the ASE in huge Affymetrix HTA 2.0 microarray datasets of blood cells from MS patients and healthy subjects. In this analysis, a subset of 19 genes was found to be differentially alternatively spliced (EventPointer $p$-value $<0.0001$ for each comparison against the 4 control datasets) in at least one of the 5 examined cell populations (CD4+, CD8+, CD14+, CD19+ and CD56+ cells). For 7 genes, the reported ASE reached statistical significance even in all case-control comparisons (Table 2).

As an example, we visualized the exon-level data of $I L 7 R$, which has been repeatedly described in the literature to be implicated in MS (Figure 3). It has been shown that skipping of exon 6 is augmented by reduced expression of the activator $D D X 39 B$ and by strengthening of an exonic splicing silencer (ESS) by the MS risk allele C of SNP rs6897932 [44, 49]. Moreover, CPSF1 has been identified to interfere with spliceosome binding of IL7R pre-mRNA [84]. As a consequence, the amount of soluble and membrane-bound protein isoforms is influenced. On HTA 2.0 microarrays, IL7R is interrogated by 245 oligonucleotide probes (probe set TC05000159.hg.1). In our data, the highest IL7R mRNA levels were seen in CD4+ and CD8+ cells. A closer inspection of the signal intensities for exon fragments and splice junctions clearly demonstrated the more frequent skipping of exon 6 in patients with MS. More specifically, we detected significantly lower average levels for exon 6 and higher levels for the junctions spanning the exon boundaries 5-7 (exon 6 skipping) and 4-7 (exon 5-6 skipping) in the MS patient group as compared to the healthy control group (Figure 3). 


\section{Discussion}

While GWAS have provided important insights into the genetic contribution to MS susceptibility [19], molecular biomarkers with high sensitivity and specificity for MS are still not available. It is likely that several MS-associated genetic variants affect regulatory regions for pre-mRNA splicing. Alterations in alternative splicing have already been demonstrated for a variety of diseases $[25,85]$. Here, we performed an electronic literature search for studies related to MS, and we reassessed the expression of splicing variants in transcriptome data of MS patients and healthy controls to further explore the biomarker potential of ASE. The article selection process was conducted according to the PRISMA guidelines [33]. For this systematic review, we screened a total of 160 articles, and we summarized the results of 36 articles that were identified as relevant to the topic. Most of the studies focused on genes that participate in inflammatory pathways or genes that encode CNS myelin proteins.

The majority of the reviewed studies examined blood cells. Blood sampling is less invasive for patients and less extensive for the medical staff compared to the collection of CSF by lumbar puncture. Nevertheless, CSF samples may be more suitable for the detection of biomarkers, as inflammatory lesions in MS selectively affect the CNS [31]. The lack of studies using CSF for the investigation of disease-specific ASE can be explained by the fact that the analysis of specimens with low cell counts was not very sensitive until a few years ago. The rapid technological development in recent years, however, enabled reliable measurements of even small amounts of RNA or protein [86-88]. Because of these advancements, it is intriguing to speculate that future studies may identify splicing products in CSF that correlate with inflammation and degeneration in MS. So far, four studies examined the expression of alternatively spliced human genes in post-mortem human brain tissue in order to better understand the pathophysiological processes in MS. For instance, different MOG isoforms have been analyzed in brain samples $[55,56]$. The findings of such studies can support and stimulate investigations of other tissues or body fluids.

The reviewed studies in the field of MS measured different RNA and protein isoforms that arise by alternative splicing and by usage of alternative transcription starts and ends. Out of 27 
reported genes, 18 genes were investigated in only one study. The currently most comprehensively studied gene is $I L 7 R$ whose isoform expression was analyzed in 8 articles. These studies revealed that the MS risk allele C of SNP rs6897932 leads to a more frequent skipping of exon 6, resulting in an increased production of transcripts that encode a soluble form of the protein [44]. Other GWAS with cohorts of primary biliary cholangitis and type 1 diabetes patients also showed an association for the rs6897932 SNP [89, 90]. Similarly, genetic variants in GSDMB appeared in GWAS on rheumatoid arthritis and ulcerative colitis [91, 92]. Furthermore, SP140 and TNFRSF1A have been implicated as risk factors in Crohn's disease and ankylosing spondylitis, respectively [93, 94]. This illustrates that the same functional mechanisms may play a role in different immune-mediated conditions that share the same genetic susceptibility loci. Further research is thus warranted to pinpoint the causal disease risk variants and to elucidate their biological consequences such as ASE.

Genotype-dependent variation in splicing regulatory elements plays a crucial role for individual splicing pattern differences, which may influence onset and outcome of chronic illnesses. Diverse bioinformatic approaches have been developed to predict such elements in silico [22, 95, 96], and splicing reporter assays can be used to evaluate the effect of genetic polymorphisms and cofactors of the spliceosome machinery in cell culture [34]. As an example, Cardamone et al. cloned IL7R exon 6 and SP140 exon 7 with different SNP alleles and with the respective intronic flanking sequences into minigene plasmids. The IL7R/SP140 minigene constructs were then cotransfected either with a MALAT1-expressing plasmid or with an empty vector into HEK 293 cells. By subsequent PCR analysis, MALAT1 overexpression was found to significantly increase skipping of IL7R exon 6 and SP140 exon 7 in a genotype-independent manner, demonstrating that this non-coding RNA affects the repertoire of alternatively spliced transcripts in MS [78]. Minigene assays have been also employed to study de novo and polymorphic Alu insertions [97]. RNA abundance and splicing are modulated by such transposable elements via RNA-protein interactions [98]. The current research on MS is still at the beginning to gain new pathomechanistic insights by assessing splicing-affecting variants and the resulting defects. Future studies should consider nucleotide diversity in larger sequence environments to better understand the context dependence of splicing regulation. 
Aberrant expression levels of alternative splicing isoforms are not only of interest for diagnostic purposes but also for monitoring therapeutic interventions in MS. Serum levels of soluble TNFRSF1A have been shown to be significantly reduced in MS patients treated with glatiramer acetate relative to untreated patients [59], and expression changes of IFNAR2 transcript isoforms in PBMC were found to be an indicator of the biological responsiveness to IFN- $\beta$ therapy [69]. A recent study by Bedri et al. explored the potential role of cytokine receptors as biomarkers for treatment effects [99]. In plasma samples from MS patients, they observed a decline in soluble $I L 7 R$ protein levels during natalizumab therapy, while subsequent fingolimod therapy led to increased levels. This is of relevance as soluble IL7R diminishes excessive IL7 signaling in human T cells and increases IL7 bioavailability [100]. Therefore, cytokines continue to be attractive therapeutic targets for neuroinflammatory disorders [101]. On the other hand, ASE may yield biomarkers of the individual clinical response to immunomodulatory treatments. However, further studies are needed to confirm and extend these findings.

One of our study eligibility criteria was the investigation of different isoforms of a human protein-coding gene. However, non-coding RNAs also contribute to transcriptomic complexity, although their regulation, spliceosomal processing and function has not been sufficiently researched so far. Several spliced transcripts can originate from human endogenous retrovirus (HERV) elements. A connection of HERV with MS has been discussed for years, but the consequences resulting from reactivated expression of retroviral sequences, which usually lack protein-coding capacity, are still unclear $[102,103]$. Christensen et al. used RNA from blood cells to detect transcripts related to the HERV-H subfamily [104]. They observed that two different HERV-H splice variants occurred together in about $40 \%$ of the MS patients but only in $10 \%$ of the controls. Another phenomenon potentially interfering with alternative splicing is represented by backsplicing of immature RNA. By this means, some spliced introns can escape degradation and generate circular RNAs [105-107]. A dysregulation of circular RNAs has been reported in MS patient blood. Cardamone et al. detected a significantly higher level of a circular RNA consisting of exons 4 and 5 of the GSDMB gene in PBMC from patients with MS compared to controls [61]. Moreover, Iparraguirre et al. carried out a microarray analysis of whole blood samples and found 406 circular RNAs to be differentially expressed in MS [108]. 
We performed a case-control reanalysis using high-density microarray datasets, which affirmed significant ASE for 19 out of the 27 examined genes. By this means, we showed that modern transcriptome profiling technology and computational biology facilitate the large-scale analysis of ASE $[43,109]$. Population transcriptome studies have fostered the identification of naturally occurring genetic variants that modulate alternative splicing [110, 111], but several challenges remain: For instance, still little is known on the contribution of RNA processing on transcript diversity of disease-associated genes of the human major histocompatibility complex (MHC) [112]. Moreover, microarray and RNA sequencing data are usually based on short sequences, thus missing information about the combination of exon junctions in individual transcripts. Emerging long-read RNA sequencing methods, however, should provide such information [113]. Individual cells can also exhibit differences in gene expression and splicing. Therefore, single-cell transcriptomics may be employed to investigate this heterogeneity [114]. Exploring the causal impacts of specific SNPs on splicing can be cumbersome. To address this issue in a high-throughput manner, massively parallel reporter assays have been designed [110]. Finally, novel proteomic profiling solutions should be incorporated to complement the characterization of dysregulated alternative splice variants [115]. The elucidation of splicing-altering disease susceptibility alleles has the potential to advance individual genomic medicine [30]. However, continued efforts are needed to improve our understanding of how ASE may affect disease onset, course and activity and the effects of immunomodulatory treatments in MS.

To conclude, we here provide an overview of published research about the expression of alternatively spliced gene isoforms in MS. Our systematic literature search identified a total of 36 studies from the last two decades, with a rising trend in recent years. Most studies used PCR techniques to measure selected genes, but first attempts have been made to incorporate transcriptome data from high-resolution microarrays and RNA sequencing. Splice variants were assessed for 27 genes, including cytokine receptors, transcription factors and myelin genes. Further studies are warranted that link genetic, RNA and protein data in a context- and cell type-specific manner to uncover splicing regulatory mechanisms. This will set the stage to gain new insights into the immunopathology of MS and to apply this knowledge in clinical practice in terms of biomarkers for disease diagnosis and prognosis as well as therapeutic decisions. 


\section{Abbreviations}

\begin{tabular}{|c|c|}
\hline ASE & alternative splicing event \\
\hline BPS & branchpoint sequence \\
\hline CNS & central nervous system \\
\hline CSF & cerebrospinal fluid \\
\hline DMD & disease-modifying drug \\
\hline ELISA & enzyme-linked immunosorbent assay \\
\hline ESE & exonic splicing enhancer \\
\hline ESS & exonic splicing silencer \\
\hline GA & glatiramer acetate \\
\hline GEO & Gene Expression Omnibus \\
\hline GWAS & genome-wide association study \\
\hline HERV & human endogenous retrovirus \\
\hline HGNC & HUGO Gene Nomenclature Committee \\
\hline HTA & Human Transcriptome Array \\
\hline ID & identifier \\
\hline IFN- $\beta$ & interferon-beta \\
\hline IMSUP & immunosuppressor \\
\hline ISE & intronic splicing enhancer \\
\hline ISS & intronic splicing silencer \\
\hline JUC & junction probe set \\
\hline $\mathrm{MHC}$ & major histocompatibility complex \\
\hline mRNA & messenger ribonucleic acid \\
\hline MS & multiple sclerosis \\
\hline n.a. & not available \\
\hline PBMC & peripheral blood mononuclear cell \\
\hline PCR & polymerase chain reaction \\
\hline PPMS & primary progressive multiple sclerosis \\
\hline PPT & polypyrimidine tract \\
\hline PRISMA & Preferred Reporting Items for Systematic Reviews and Meta-Analyses \\
\hline PSR & probe selection region probe set \\
\hline RNA & ribonucleic acid \\
\hline RRMS & relapsing-remitting multiple sclerosis \\
\hline
\end{tabular}


SNP single-nucleotide polymorphism

SPMS secondary progressive multiple sclerosis

SST-RMA signal space transformation robust multi-array average

TAC Transcriptome Analysis Console

Th cell Thelper cell

Treg regulatory $\mathrm{T}$ cell 


\section{Acknowledgments}

NB is a scholarship holder of the Stiftung der Deutschen Wirtschaft (sdw). The microarray analysis was partially funded by Novartis Pharma and Genzyme. The funders had no role in the study design, interpretation of data, decision to publish or preparation of the manuscript.

\section{Disclosures}

$\mathrm{MH}$ has received speaking fees and travel support from Bayer, Biogen, Novartis and Teva. NB has received travel support from Novartis. PSR has received research grants, travel support, advisory fees and/or honoraria from Biogen, Daiichi Sankyo, Genzyme, Merck, Novartis, Roche, Shire and Teva. UKZ has received speaking fees, travel support and/or financial support for research activities from Almirall, Bayer, Biogen, Merck Serono, Novartis, Sanofi Genzyme and Teva. 


\section{References}

[1] Filippi M, Bar-Or A, Piehl F, Preziosa P, Solari A, Vukusic S, et al. Multiple sclerosis. Nat Rev Dis Primers 2018;4(1):43.

[2] Browne P, Chandraratna D, Angood C, Tremlett H, Baker C, Taylor BV, et al. Atlas of Multiple Sclerosis 2013: A growing global problem with widespread inequity. Neurology 2014;83(11):1022-4.

[3] Goodin DS. The epidemiology of multiple sclerosis: insights to a causal cascade. Handb Clin Neurol 2016;138:173-206.

[4] Lublin FD, Reingold SC, Cohen JA, Cutter GR, Sørensen PS, Thompson AJ, et al. Defining the clinical course of multiple sclerosis: the 2013 revisions. Neurology 2014;83(3):278-86.

[5] Zettl UK, Stüve O, Patejdl R. Immune-mediated CNS diseases: a review on nosological classification and clinical features. Autoimmun Rev 2012;11(3):167-73.

[6] Tremlett H, Yinshan Zhao, Devonshire V. Natural history of secondary-progressive multiple sclerosis. Mult Scler 2008;14(3):314-24.

[7] Galea I, Ward-Abel N, Heesen C. Relapse in multiple sclerosis. BMJ 2015;350:h1765.

[8] Ellenberger D, Eichstädt K, Flachenecker P, Friede T, Haas J, Kleinschnitz C, et al. Decreasing longitudinal use of glucocorticosteroids in multiple sclerosis. Mult Scler Relat Disord 2018;25:173-4.

[9] Skierlo S, Rommer PS, Zettl UK. Symptomatic treatment in multiple sclerosis-interim analysis of a nationwide registry. Acta Neurol Scand 2017;135(4):394-9.

[10] Giovannoni G. Disease-modifying treatments for early and advanced multiple sclerosis: a new treatment paradigm. Curr Opin Neurol 2018;31(3):233-43.

[11] Zettl UK, Hecker M, Aktas O, Wagner T, Rommer PS. Interferon $\beta-1 \mathrm{a}$ and $\beta-1 \mathrm{~b}$ for patients with multiple sclerosis: updates to current knowledge. Expert Rev Clin Immunol 2018;14(2):137-53.

[12] Derfuss T, Ontaneda D, Nicholas J, Meng X, Hawker K. Relapse rates in patients with multiple sclerosis treated with fingolimod: Subgroup analyses of pooled data from three phase 3 trials. Mult Scler Relat Disord 2016;8:124-30.

[13] Montalban X, Hauser SL, Kappos L, Arnold DL, Bar-Or A, Comi G, et al. Ocrelizumab versus Placebo in Primary Progressive Multiple Sclerosis. N Engl J Med 2017;376(3):20920.

[14] Ascherio A, Munger KL. Epidemiology of Multiple Sclerosis: From Risk Factors to Prevention-An Update. Semin Neurol 2016;36(2):103-14.

[15] Pierrot-Deseilligny C, Souberbielle JC. Vitamin D and multiple sclerosis: An update. Mult Scler Relat Disord 2017;14:35-45.

[16] Olsson T, Barcellos LF, Alfredsson L. Interactions between genetic, lifestyle and 
environmental risk factors for multiple sclerosis. Nat Rev Neurol 2017;13(1):25-36.

[17] Westerlind H, Ramanujam R, Uvehag D, Kuja-Halkola R, Boman M, Bottai M, et al. Modest familial risks for multiple sclerosis: a registry-based study of the population of Sweden. Brain 2014;137(Pt 3):770-8.

[18] O'Gorman C, Lin R, Stankovich J, Broadley SA. Modelling genetic susceptibility to multiple sclerosis with family data. Neuroepidemiology 2013;40(1):1-12.

[19] Patsopoulos NA, Baranzini SE, Santaniello A, Shoostari P, Cotsapas C, Wong G, et al. The Multiple Sclerosis Genomic Map: Role of peripheral immune cells and resident microglia in susceptibility. bioRxiv 143933; doi: https://doi.org/10.1101/143933.

[20] Lee Y, Rio DC. Mechanisms and Regulation of Alternative Pre-mRNA Splicing. Annu Rev Biochem 2015;84:291-323.

[21] Manning KS, Cooper TA. The roles of RNA processing in translating genotype to phenotype. Nat Rev Mol Cell Biol 2017;18(2):102-14.

[22] Anna A, Monika G. Splicing mutations in human genetic disorders: examples, detection, and confirmation. J Appl Genet 2018;59(3):253-68.

[23] Pineda JMB, Bradley RK. Most human introns are recognized via multiple and tissuespecific branchpoints. Genes Dev 2018;32(7-8):577-91.

[24] Fu XD, Ares M Jr. Context-dependent control of alternative splicing by RNA-binding proteins. Nat Rev Genet 2014;15(10):689-701.

[25] Scotti MM, Swanson MS. RNA mis-splicing in disease. Nat Rev Genet 2016;17(1):19-32.

[26] Levin AA. Treating Disease at the RNA Level with Oligonucleotides. N Engl J Med 2019;380(1):57-70.

[27] Goodwin S, McPherson JD, McCombie WR. Coming of age: ten years of next-generation sequencing technologies. Nat Rev Genet 2016;17(6):333-51.

[28] Gonzalo R, Sánchez A. Introduction to Microarrays Technology and Data Analysis. In: Jaumot J, Bedia C, Tauler R, editors. Data Analysis for Omic Sciences: Methods and Applications, Amsterdam: Elsevier; 2018, p. 37-69.

[29] Li YI, van de Geijn B, Raj A, Knowles DA, Petti AA, Golan D, et al. RNA splicing is a primary link between genetic variation and disease. Science 2016;352(6285):600-4 .

[30] Wai H, Douglas AGL, Baralle D. RNA splicing analysis in genomic medicine. Int J Biochem Cell Biol 2019;108:61-71.

[31] Fitzner B, Hecker M, Zettl UK. Molecular biomarkers in cerebrospinal fluid of multiple sclerosis patients. Autoimmun Rev 2015;14(10):903-13.

[32] Comabella M, Montalban X. Body fluid biomarkers in multiple sclerosis. Lancet Neurol 2014;13(1):113-26.

[33] Liberati A, Altman DG, Tetzlaff J, Mulrow C, Gøtzsche PC, loannidis JP, et al. The PRISMA statement for reporting systematic reviews and meta-analyses of studies that evaluate 
health care interventions: explanation and elaboration. PLoS Med 2009;6(7):e1000100.

[34] Gaildrat P, Killian A, Martins A, Tournier I, Frébourg T, Tosi M. Use of splicing reporter minigene assay to evaluate the effect on splicing of unclassified genetic variants. Methods Mol Biol 2010;653:249-57.

[35] Stelzer G, Rosen N, Plaschkes I, Zimmerman S, Twik M, Fishilevich S, et al. The GeneCards Suite: From Gene Data Mining to Disease Genome Sequence Analyses. Curr Protoc Bioinformatics 2016;54:1.30.1-33.

[36] UniProt Consortium. UniProt: a worldwide hub of protein knowledge. Nucleic Acids Res 2019;47(D1):D506-15.

[37] Cunningham F, Achuthan P, Akanni W, Allen J, Amode MR, Armean IM, et al. Ensembl 2019. Nucleic Acids Res 2019;47(D1):D745-51.

[38] Roch L, Hecker M, Friess J, Angerer IC, Koczan D, Fitzner B, et al. High-Resolution Expression Profiling of Peripheral Blood CD8+ Cells in Patients with Multiple Sclerosis Displays Fingolimod-Induced Immune Cell Redistribution. Mol Neurobiol 2017;54(7):551125.

[39] Friess J, Hecker M, Roch L, Koczan D, Fitzner B, Angerer IC, et al. Fingolimod alters the transcriptome profile of circulating CD4+ cells in multiple sclerosis. Sci Rep 2017;7:42087.

[40] Angerer IC, Hecker M, Koczan D, Roch L, Friess J, Rüge A, et al. Transcriptome profiling of peripheral blood immune cell populations in multiple sclerosis patients before and during treatment with a sphingosine-1-phosphate receptor modulator. CNS Neurosci Ther 2018;24(3):193-201.

[41] Koczan D, Fitzner B, Zettl UK, Hecker M. Microarray data of transcriptome shifts in blood cell subsets during S1P receptor modulator therapy. Sci Data 2018;5:180145.

[42] Hoffman RW, Merrill JT, Alarcón-Riquelme MM, Petri M, Dow ER, Nantz E, et al. Gene Expression and Pharmacodynamic Changes in 1,760 Systemic Lupus Erythematosus Patients From Two Phase III Trials of BAFF Blockade With Tabalumab. Arthritis Rheumatol 2017;69(3):643-54.

[43] Romero JP, Muniategui A, De Miguel FJ, Aramburu A, Montuenga L, Pio R, et al. EventPointer: an effective identification of alternative splicing events using junction arrays. BMC Genomics 2016;17:467.

[44] Gregory SG, Schmidt S, Seth P, Oksenberg JR, Hart J, Prokop A, et al. Interleukin 7 receptor alpha chain (IL7R) shows allelic and functional association with multiple sclerosis. Nat Genet 2007;39(9):1083-91.

[45] McKay FC, Swain LI, Schibeci SD, Rubio JP, Kilpatrick TJ, Heard RN, et al. Haplotypes of the interleukin 7 receptor alpha gene are correlated with altered expression in whole blood cells in multiple sclerosis. Genes Immun 2008;9(1):1-6.

[46] Rane L, Vudattu N, Bourcier K, Graniar E, Hillert J, Seyfert V, et al. Alternative splicing of interleukin-7 (IL-7) and interleukin-7 receptor alpha (IL-7Ralpha) in peripheral blood from patients with multiple sclerosis (MS). J Neuroimmunol 2010;222(1-2):82-6.

[47] Hoe E, McKay FC, Schibeci SD, Gandhi K, Heard RN, Stewart GJ, et al. Functionally significant differences in expression of disease-associated IL-7 receptor alpha haplotypes 
in CD4 T cells and dendritic cells. J Immunol 2010;184(5):2512-7.

[48] McKay FC, Hoe E, Parnell G, Gatt P, Schibeci SD, Stewart GJ, et al. IL7Ra expression and upregulation by IFN $\beta$ in dendritic cell subsets is haplotype-dependent. PLoS One 2013;8(10):e77508.

[49] Galarza-Muñoz G, Briggs FBS, Evsyukova I, Schott-Lerner G, Kennedy EM, Nyanhete T, et al. Human Epistatic Interaction Controls IL7R Splicing and Increases Multiple Sclerosis Risk. Cell 2017;169(1):72-84.

[50] Sharief MK. Increased cellular expression of the caspase inhibitor FLIP in intrathecal lymphocytes from patients with multiple sclerosis. J Neuroimmunol 2000;111(1-2):203-9.

[51] Semra YK, Seidi OA, Sharief MK. Overexpression of the apoptosis inhibitor FLIP in T cells correlates with disease activity in multiple sclerosis. J Neuroimmunol 2001;113(2):268-74.

[52] Seidi OA, Sharief MK. The expression of apoptosis-regulatory proteins in B lymphocytes from patients with multiple sclerosis. J Neuroimmunol 2002;130(1-2):202-10.

[53] Jacobsen M, Schweer D, Ziegler A, Gaber R, Schock S, Schwinzer R, et al. A point mutation in PTPRC is associated with the development of multiple sclerosis. Nat Genet 2000;26(4):495-9.

[54] Jacobsen M, Hoffmann S, Cepok S, Stei S, Ziegler A, Sommer N, et al. A novel mutation in PTPRC interferes with splicing and alters the structure of the human CD45 molecule. Immunogenetics 2002;54(3):158-63.

[55] Boyle LH, Traherne JA, Plotnek G, Ward R, Trowsdale J. Splice variation in the cytoplasmic domains of myelin oligodendrocyte glycoprotein affects its cellular localisation and transport. J Neurochem 2007;102(6):1853-62.

[56] Jensen CJ, Stankovich J, Butzkueven H, Oldfield BJ, Rubio JP. Common variation in the MOG gene influences transcript splicing in humans. J Neuroimmunol 2010;229(1-2):22531.

[57] Carbone F, De Rosa V, Carrieri PB, Montella S, Bruzzese D, Porcellini A, et al. Regulatory $T$ cell proliferative potential is impaired in human autoimmune disease. Nat Med 2014;20(1):69-74.

[58] De Rosa V, Galgani M, Porcellini A, Colamatteo A, Santopaolo M, Zuchegna C, et al. Glycolysis controls the induction of human regulatory $T$ cells by modulating the expression of FOXP3 exon 2 splicing variants. Nat Immunol 2015;16(11):1174-84.

[59] Ottoboni L, Frohlich IY, Lee M, Healy BC, Keenan BT, Xia Z, et al. Clinical relevance and functional consequences of the TNFRSF1A multiple sclerosis locus. Neurology 2013;81(22):1891-9.

[60] Gregory AP, Dendrou CA, Attfield KE, Haghikia A, Xifara DK, Butter F, et al. TNF receptor 1 genetic risk mirrors outcome of anti-TNF therapy in multiple sclerosis. Nature 2012;488(7412):508-11.

[61] Cardamone G, Paraboschi EM, Rimoldi V, Duga S, Soldà G, Asselta R. The Characterization of GSDMB Splicing and Backsplicing Profiles Identifies Novel Isoforms and a Circular RNA That Are Dysregulated in Multiple Sclerosis. Int J Mol Sci 
[62] De Rossi P, Buggia-Prévot V, Clayton BL, Vasquez JB, van Sanford C, Andrew RJ, et al. Predominant expression of Alzheimer's disease-associated BIN1 in mature oligodendrocytes and localization to white matter tracts. Mol Neurodegener 2016;11(1):59.

[63] Paraboschi EM, Cardamone G, Rimoldi V, Gemmati D, Spreafico M, Duga S, et al. MetaAnalysis of Multiple Sclerosis Microarray Data Reveals Dysregulation in RNA Splicing Regulatory Genes. Int J Mol Sci 2015;16(10):23463-81.

[64] Matesanz F, Potenciano V, Fedetz M, Ramos-Mozo P, Abad-Grau Mdel M, Karaky M, et al. A functional variant that affects exon-skipping and protein expression of SP140 as genetic mechanism predisposing to multiple sclerosis. Hum Mol Genet 2015;24(19):561927.

[65] Spurlock CF 3rd, Tossberg JT, Guo Y, Sriram S, Crooke PS 3rd, Aune TM. Defective structural RNA processing in relapsing-remitting multiple sclerosis. Genome Biol 2015;16:58.

[66] Paraboschi EM, Rimoldi V, Soldà G, Tabaglio T, Dall'Osso C, Saba E, et al. Functional variations modulating PRKCA expression and alternative splicing predispose to multiple sclerosis. Hum Mol Genet 2014;23(25):6746-61.

[67] Tian Y, Apperson ML, Ander BP, Liu D, Stomova BS, Jickling GC, et al. Differences in exon expression and alternatively spliced genes in blood of multiple sclerosis compared to healthy control subjects. J Neuroimmunol 2011;230(1-2):124-9.

[68] Palacios R, Comas D, Elorza J, Villoslada P. Genomic regulation of CTLA4 and multiple sclerosis. J Neuroimmunol 2008;203(1):108-15.

[69] Gilli F, Valentino P, Caldano M, Granieri L, Capobianco M, Malucchi S, et al. Expression and regulation of IFNalpha/beta receptor in IFNbeta-treated patients with multiple sclerosis. Neurology 2008;71(24):1940-7.

[70] Astier AL, Meiffren G, Freeman S, Hafler DA. Alterations in CD46-mediated Tr1 regulatory T cells in patients with multiple sclerosis. J Clin Invest 2006;116(12):3252-7.

[71] Johnston JB, Silva C, Gonzalez G, Holden J, Warren KG, Metz LM, et al. Diminished adenosine $A 1$ receptor expression on macrophages in brain and blood of patients with multiple sclerosis. Ann Neurol 2001;49(5):650-8.

[72] Sestito C, Brevé JJP, Killestein J, Teunissen CE, Wilhelmus MMM, Drukarch B, et al. Differential Expression of Tissue Transglutaminase Splice Variants in Peripheral Blood Mononuclear Cells of Primary Progressive Multiple Sclerosis Patients. Med Sci (Basel) 2018;6(4):108.

[73] Uzawa A, Mori M, Hayakawa S, Masuda S, Nomura F, Kuwabara S. Expression of chemokine receptors on peripheral blood lymphocytes in multiple sclerosis and neuromyelitis optica. BMC Neurol 2010;10:113.

[74] López-Gómez C, Oliver-Martos B, Pinto-Medel MJ, Suardiaz M, Reyes-Garrido V, Urbaneja $P$, et al. TRAIL and TRAIL receptors splice variants during long-term interferon $\beta$ treatment of patients with multiple sclerosis: evaluation as biomarkers for therapeutic response. J Neurol Neurosurg Psychiatry 2016;87(2):130-7. 
[75] Field J, Shahijanian F, Schibeci S; Australia and New Zealand MS Genetics Consortium (ANZgene), Johnson L, Gresle M, et al. The MS Risk Allele of CD40 Is Associated with Reduced Cell-Membrane Bound Expression in Antigen Presenting Cells: Implications for Gene Function. PLoS One 2015;10(6):e0127080.

[76] Smets I, Fiddes B, Garcia-Perez JE, He D, Mallants K, Liao W, et al. Multiple sclerosis risk variants alter expression of co-stimulatory genes in B cells. Brain 2018;141(3):786-96.

[77] Sambucci M, Gargano F, De Rosa V, De Bardi M, Picozza M, Placido R, et al. FoxP3 isoforms and PD-1 expression by T regulatory cells in multiple sclerosis. Sci Rep 2018;8(1):3674.

[78] Cardamone G, Paraboschi EM, Soldà G, Cantoni C, Supino D, Piccio L, et al. Not only cancer: the long non-coding RNA MALAT1 affects the repertoire of alternatively spliced transcripts and circular RNAs in multiple sclerosis. Hum Mol Genet 2019. doi: 10.1093/hmg/ddy438.

[79] Booth DR, Arthur AT, Teutsch SM, Bye C, Rubio J, Armati PJ, et al. Gene expression and genotyping studies implicate the interleukin 7 receptor in the pathogenesis of primary progressive multiple sclerosis. J Mol Med (Berl) 2005;83(10):822-30.

[80] Reindl M, Waters P. Myelin oligodendrocyte glycoprotein antibodies in neurological disease. Nat Rev Neurol 2019;15(2):89-102.

[81] Karaky M, Fedetz M, Potenciano V, Andrés-León E, Codina AE, Barrionuevo C, et al. SP140 regulates the expression of immune-related genes associated with multiple sclerosis and other autoimmune diseases by NF-kB inhibition. Hum Mol Genet 2018;27(23):4012-23.

[82] Kleinewietfeld M, Manzel A, Titze J, Kvakan H, Yosef N, Linker RA, et al. Sodium chloride drives autoimmune disease by the induction of pathogenic TH17 cells. Nature 2013;496(7446):518-22.

[83] Wang H, Ferraris JD, Klein JD, Sands JM, Burg MB, Zhou X. PKC- $\alpha$ contributes to high $\mathrm{NaCl}$-induced activation of NFAT5 (TonEBP/OREBP) through MAPK ERK1/2. Am J Physiol Renal Physiol 2015;308(2):F140-8.

[84] Evsyukova I, Bradrick SS, Gregory SG, Garcia-Blanco MA. Cleavage and polyadenylation specificity factor 1 (CPSF1) regulates alternative splicing of interleukin 7 receptor (IL7R) exon 6. RNA 2013;19(1):103-15.

[85] Evsyukova I, Somarelli JA, Gregory SG, Garcia-Blanco MA. Alternative splicing in multiple sclerosis and other autoimmune diseases. RNA Biol 2010;7(4):462-73.

[86] Quan PL, Sauzade M, Brouzes E. dPCR: A Technology Review. Sensors (Basel) 2018;18(4):1271.

[87] Cohen L, Walt DR. Single-Molecule Arrays for Protein and Nucleic Acid Analysis. Annu Rev Anal Chem (Palo Alto Calif) 2017;10(1):345-63.

[88] Lycke J, Zetterberg H. The role of blood and CSF biomarkers in the evaluation of new treatments against multiple sclerosis. Expert Rev Clin Immunol 2017;13(12):1143-53.

[89] Kawashima M, Hitomi Y, Aiba Y, Nishida N, Kojima K, Kawai Y, et al. Genome-wide association studies identify PRKCB as a novel genetic susceptibility locus for primary 

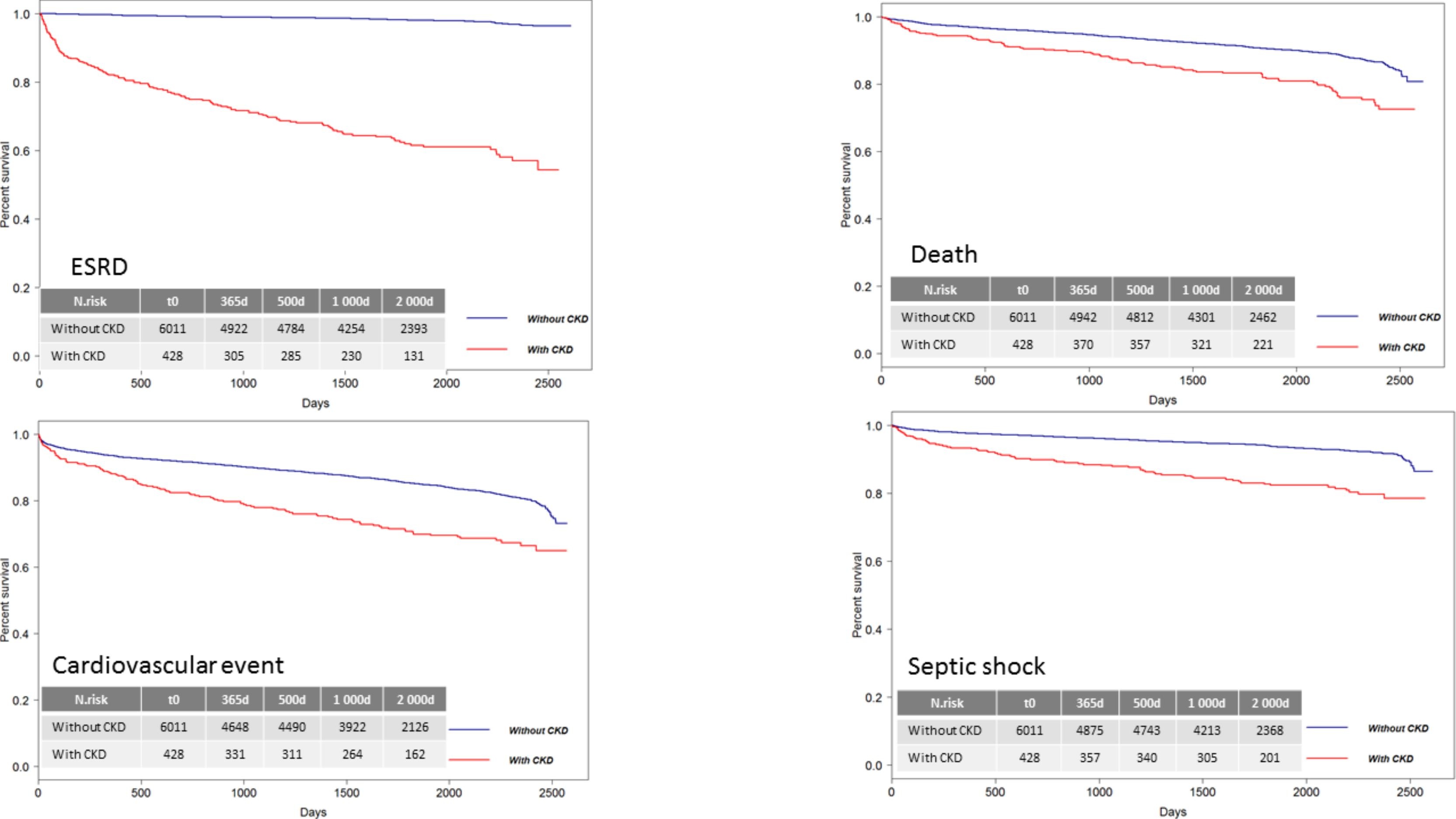